SCIENTIFIC LETTER

\title{
Central $\alpha_{2}$ adrenoceptors and the pathogenesis of carotid sinus hypersensitivity
}

\section{S W Parry, M Baptist, J J Gilroy, N Steen, R A Kenny}

Heart 2004;90:935-936. doi: 10.1136/hrt.2002.006205

W hile the physiology of the normal carotid baroreflex is reasonably well established, the pathophysiology of carotid sinus hypersensitivity $(\mathrm{CSH})$ remains obscure. It has been proposed that central $\alpha_{2}$ adrenoceptor upregulation provides the substrate for the changes in baroreflex gain which manifest as $\mathrm{CSH}^{1}{ }^{1}$ This hypothesis suggests that carotid sinus stiffness resulting from age related cardiovascular disease causes relative diminution of afferent baroreceptor neural traffic, with compensatory brain stem post-synaptic $\alpha_{2}$ adrenoceptor upregulation. This physiologic denervation hypersensitivity then causes the overshoot bradycardia and hypotension following carotid sinus stimulation that is clinical CSH. Though widely quoted, this hypothesis has no evidence base, and no attempts have been made to date to test it. If $\alpha_{2}$ adrenoceptor hypersensitivity was the major pathophysiological defect in CSH, a centrally active $\alpha_{2}$ adrenoceptor antagonist should abolish or attenuate the effects of carotid sinus massage (CSM) in such individuals. ${ }^{12}$ In order to test this hypothesis we studied the effects of the central $\alpha_{2}$ adrenoceptor antagonist yohimbine ${ }^{3}$ on the vasodepressor response to CSM in syncopal subjects with $\mathrm{CSH}$, in a randomised, double blind, placebo controlled study, utilising a crossover design.

\section{METHODS}

Eighteen consecutive patients with syncope caused by CSH, who had been referred for permanent pacemaker implantation through our syncope facility and had a minimum reproducible vasodepressor response of $20 \mathrm{~mm} \mathrm{Hg}$ during CSM post-pacemaker, participated in this study. While a $50 \mathrm{~mm} \mathrm{Hg}$ fall in systolic blood pressure (SBP) during CSM defines vasodepressor $\mathrm{CSH}_{1}{ }^{4}$ a previous study has shown that during supine CSM in healthy subjects, mean vasodepression was $19 \mathrm{~mm} \mathrm{Hg}$ on the right, and $14 \mathrm{~mm} \mathrm{Hg}$ on the left. 5 Subjects were studied post-pacemaker implantation so that the fixed cardiac pacing rate avoided the confounding effects of hypotension secondary to variable bradycardic or asystolic

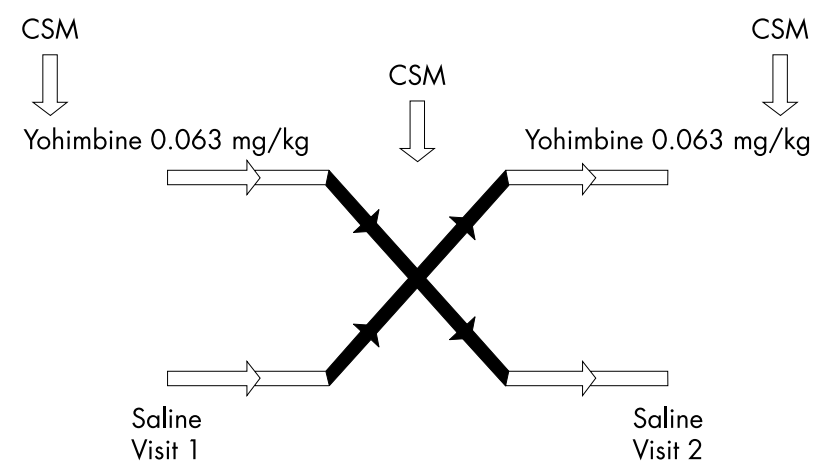

Figure 1 Central $\alpha_{2}$ adrenoceptor hypothesis: study design. CSM, carotid sinus massage.

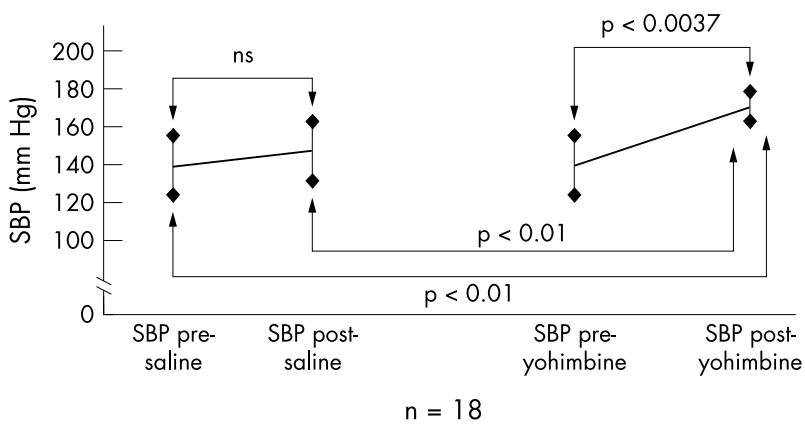

Figure 2 Change in systolic blood pressure (SBP) post-intravenous yohimbine and saline.

responses to CSM. The investigation had local ethical approval. Following informed, written consent, $10 \mathrm{ml}$ solutions of intravenous yohimbine hydrochloride $(0.063 \mathrm{mg} / \mathrm{kg})$ and normal saline were infused over two minutes into each subject via antecubital cannulae, on separate days, in random, double blind fashion with a minimum 48 hours between injections to ensure adequate washout of the active drug (distribution half life 0.4-18 minutes, elimination halflife 15-150 minutes) (fig 1). ${ }^{3}$

Twelve lead ECG and non-invasive beat-to-beat blood pressure measurements (Finapres, Ohmeda, Wisconsin, USA) were then recorded at baseline and during supine, sequential, bilateral and longitudinal CSM (with a one minute interval between episodes) for five seconds before and after each injection. Continuous ECG and blood pressure monitoring occurred throughout the study periods. Time to SBP nadir with CSM was also recorded. The mean differences in vasodepressor response and time to SBP nadir following bilateral CSM before and after saline injection were compared to differences before and after yohimbine injection using an independent sample $t$ test.

\section{RESULTS}

Of 18 subjects, nine were female and the mean age was 73.9 (8.31) years. None of the subjects were on medications that interacted with $\alpha_{2}$ adrenoceptors or yohimbine. Seven (39\%) subjects experienced side effects following yohimbine infusion which included tremulousness, anxiety, teeth chattering, and cold sweat; one subject experienced a headache after receiving the placebo. The rise in SBP following yohimbine injection ( $37 \mathrm{~mm} \mathrm{Hg}$ ) was significantly greater than that following saline injection $(15 \mathrm{~mm} \mathrm{Hg}, 95 \%$ confidence interval (CI) 8.12 to 35.54; $\mathrm{p}=0.0037$ ), a difference of $22 \mathrm{~mm} \mathrm{Hg}$ (fig 2). Vasodepressor responses and time to SBP

Abbreviations: $\mathrm{CSH}$, carotid sinus hypersensitivity; $\mathrm{CSM}$, carotid sinus massage; SBP, systolic blood pressure 
Table 1 Vasodepressor response and time to SBP nadir following CSM with saline or yohimbine injection

\begin{tabular}{|c|c|c|c|c|c|c|}
\hline \multirow[b]{2}{*}{ Drug } & \multicolumn{3}{|c|}{ Vasodepressor responses to CSM $(\mathrm{mm} \mathrm{Hg})$} & \multicolumn{3}{|c|}{ Mean time to SBP nadir (seconds) } \\
\hline & Pre-injection & Post-injection & Difference & Pre-injection & Post-injection & Difference \\
\hline $\begin{array}{l}\text { Saline } \\
\mathrm{n}=18 \\
\text { Yohimbine } \\
\mathrm{n}=18\end{array}$ & $\begin{array}{l}22.22 \\
(16.03 \text { to } 28.42) \\
25.83 \\
(20.96 \text { to } 30.70)\end{array}$ & $\begin{array}{l}17.50 \\
(12.10 \text { to } 22.90) \\
22.80 \\
(17.58 \text { to } 28.03)\end{array}$ & $\begin{array}{l}4.72 \\
(-2.34 \text { to } 1.79) \\
3.03 \\
(-2.76 \text { to } 8.82)\end{array}$ & $\begin{array}{l}13.58 \\
(12.28 \text { to } 14.89) \\
13.86 \\
(12.23 \text { to } 15.49)\end{array}$ & $\begin{array}{l}12.62 \\
(11.07 \text { to } 14.17) \\
14.19 \\
(12.21 \text { to } 16.17)\end{array}$ & $\begin{array}{l}0.77 \\
(1.13 \text { to } 2.67) \\
-0.48 \\
(-3.12 \text { to } 2.16)\end{array}$ \\
\hline
\end{tabular}

nadir with CSM are detailed in table 1 and fig 3 (vasodepressor responses only).

The mean change in vasodepressor response to CSM before and after saline injection was $4.72 \mathrm{~mm} \mathrm{Hg} \mathrm{(95 \%} \mathrm{CI}-2.34$ to 11.79), while that before and after yohimbine injection was $3.03 \mathrm{~mm} \mathrm{Hg}$ (95\% CI -2.76 to 8.82 ) (table 1, fig 3). This difference (1.69 $\mathrm{mm} \mathrm{Hg}$ ) was not significant (95\% CI -8.88 to $12.27, \mathrm{p}=0.725)$. There was no significant difference in the time to SBP nadir following yohimbine and saline injection (difference 1.25 seconds, 95\% CI -1.98 to 4.48 , $\mathrm{p}=0.44)($ table 1$)$.

\section{DISCUSSION}

We found no evidence of a critical role for central $\alpha_{2}$ adrenoceptor upregulation in the pathogenesis of CSH. If the hypothesis were correct, the powerful central $\alpha_{2}$ adrenoceptor antagonist yohimbine should have attenuated the vasodepressor response to CSM; it did not. Criticism could be levelled at the small vasodepressor response (less than the usual SBP criteria for vasodepressor CSH), but its previous validation as an abnormal response, ${ }^{5}$ reproducibility and the within subjects control factor afforded by the crossover design, make this unlikely. Furthermore, the use of patients with a pure vasodepressor response to CSM (that is $>50 \mathrm{~mm} \mathrm{Hg}$ fall in SBP with CSM) may have increased the risk of watershed type neurological complications, particularly given the repeated episodes of CSM in each

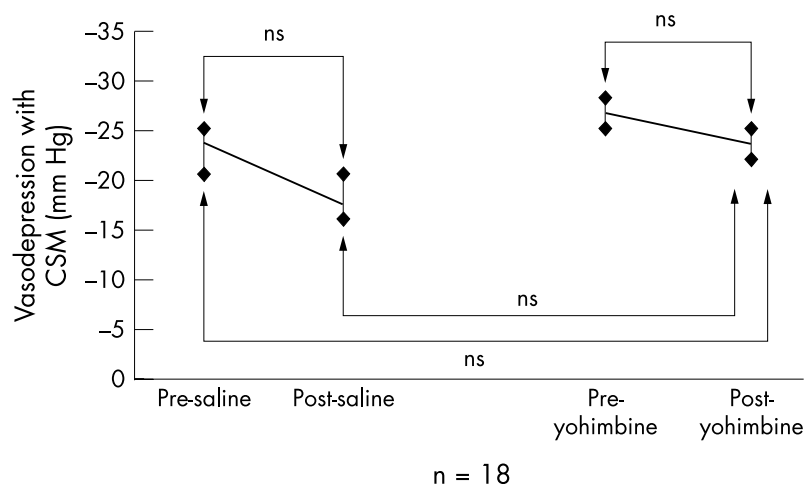

Figure 3 Vasodepressor response with carotid sinus massage (CSM) before and after saline or yohimbine injection. patient. Patients with a pacemaker already implanted, controlled for the potentially confounding effects of bradycardia/asystole on blood pressure. O'Mahony suggested premedication with apraclonidine before yohimbine injection to ensure only central $\alpha_{2}$ adrenoceptor blockade. ${ }^{1}$ While the hypertension and other hyperadrenergic side effects experienced by study participants clearly demonstrate the adequacy of central $\alpha_{2}$ adrenoceptor blockade, the vasodepressor response to CSM during yohimbine administration was unchanged, arguing strongly against a significant role for these receptors in CSH.

The $\alpha_{2}$ adrenoceptor hypothesis, though plausible, remains speculative and without evidential foundations. Adequately powered, replicative studies are needed, as are more detailed neurohistochemical and neuropathological studies. Therapeutic strategies in any disease should ideally be guided by a sound understanding of its pathophysiology; while permanent cardiac pacing is effective in the management of cardioinhibition in $\mathrm{CSH}$, there is no adequate treatment for the vasodepressor component of the disorder. Further work on the underlying pathophysiology of CSH is thus not simply an academic exercise but a clinical imperative.

\section{Authors' affiliations}

S W Parry, M Baptist, J J Gilroy, I N Steen, R A Kenny, Cardiovascular Investigation Unit and Institute for the Health of the Elderly, Royal

Victoria Infirmary, Newcastle upon Tyne, UK

Correspondence to: Dr Steve W Parry, Cardiovascular Investigation Unit and Institute for the Health of the Elderly, c/o Ward 15 Offices, Royal Victoria Infirmary, Newcastle upon Tyne, UK; swparry@ hotmail.com

Accepted 29 May 2003

\section{REFERENCES}

O'Mahony D. Pathophysiology of carotid sinus hypersensitivity in elderly patients. Lancet 1995:346:950-2.

2 Goldberg MR, Robertson D. Yohimbine: A pharmacological probe for study of the alpha-2 adrenoceptor. Pharmacol Rev 1983;35:143-80.

3 Dollery C. Yohimbine (hydrochloride). In: Dollery C, ed. Therapeutic drugs, $2^{\text {nd }}$ ed. Edinburgh: Churchill Livingstone, 1999.

4 Kenny RA, O'Shea D, Parry SW. The Newcastle protocols for head-up tilt table testing in the diagnosis of vasovagal syncope, carotid sinus hypersensitivity, and related disorders. Heart 2000;83:564-9.

5 Mclntosh SJ, Lawson J, Kenny RA. Heart rate and blood pressure responses to carotid sinus massage in healthy elderly subjects. Age Ageing 1994;23:57-61 . 